\section{The 34th Annual Meeting of the J.B. Johnston Club for Evolutionary Neuroscience and the 26th Annual Karger Workshop in Evolutionary Neuroscience}

The 2014 meetings of the J.B. Johnston Club for Evolutionary Neuroscience and Karger Workshop in Evolutionary Neuroscience will be held immediately before the annual meeting of the Society for Neuroscience on Thursday, November 13 (the Karger Workshop), and Friday, November 14 (the regular JBJC meeting). Both meetings will take place at the Embassy Row Hotel in Washington, D.C., USA.

This year's Karger Workshop, made possible by the continuing support of Karger Publishers, is co-organized by Elizabeth and Kenneth Catania. It is titled 'The Neurobiology and Behavior of Predators and Prey'. The Workshop features eight invited talks by speakers who will review diverse adaptations that make predators more adept at catching prey, and the prey more capable of escaping or defending itself. On the following day, the program for annual JBJC meeting will consist of 17 talks submitted by JBJC members selected by the JBJC Program Committee (Michael Pritz, Chris Braun, and Bruce Carlson) plus a presentation by this year's invited Karger Speaker, Dr. Baldomero Olivera. The meeting also includes a 'data blitz' of very brief presentations. Additional information and the final schedule of talks will be mailed to JBJC members before the meeting and posted on the JBJC web site (www. jbjclub.org).

\section{Karger Workshop in Evolutionary Neuroscience: The Neurobiology and Behavior of Predators and Prey}

\section{Organized by: Elizabeth Catania and Kenneth Catania (Vanderbilt University, Nashville, Tenn., USA)}

Speakers giving presentations at the 2014 Karger Workshop in Evolutionary Neuroscience are listed below. The final schedule of talks will be sent to the membership prior to the meeting and will be available at the registration desk during the meeting.

- Cynthia Moss, Johns Hopkins University, College Park, Md., USA

- Catherine Carr, University of Maryland, College Park, Md., USA
- Paloma T. Gonzalez-Bellido, University of Cambridge, Cambridge, UK

- Malcolm A. MacIver, Northwestern University, Evanston, Ill., USA

- Kenneth C. Catania, Vanderbilt University, Nashville, Tenn., USA

- Ashlee Rowe, Michigan State University, East Lansing, Mich., USA

- Edmund D. Brodie III, University of Virginia, Charlottesville, Va., USA

- Baldomero Olivera, University of Utah, Salt Lake City, Utah, USA

\section{J.B. Johnston Club for Evolutionary Neuroscience Meeting Abstracts}

Abstracts for talks scheduled for the 2014 annual meeting of the J.B. Johnston Club for Evolutionary Neuroscience are listed in alphabetical order by presenting author. The final schedule of talks will be sent to the membership prior to the meeting and will be available at the registration desk during the meeting. This year's Karger Invited Guest will be Dr. Baldomero Olivera. The title of his talk will be: 'Using Fish-Hunting Cone Snails to Understand Nervous System Complexity'.

\section{Evolutionary Divergence in Peripheral Sensory Coding Strategies in Mormyrid Weakly Electric Fishes \\ Baker CA, Huck K, Carlson BA \\ Department of Biology, Washington University in St. Louis, St. Louis, Mo., USA \\ cabaker@wustl.edu}

In mormyrid weakly electric fishes, enhanced perceptual abilities have been linked to the evolution of brain regions that process electric communication signals. Electric communication in mormyrids consists of a brief electric organ discharge (EOD) produced at variable interpulse intervals. The EOD waveform can carry identifying information such as species and sex, and the sequence of intervals conveys behavioral state. Some mormyrid species can detect small differences in EOD waveforms, whereas others cannot.
2014 S. Karger AG, Basel

0006-8977/14/0841-0066\$39.50/0 
An electrosensory midbrain region that processes communication signals is enlarged and subdivided in species that can detect EOD waveform variation. Furthermore, species sensitive to EOD waveform differences have peripheral sensory receptors that are distributed across the head, back, and underbelly, whereas species insensitive to EOD variation have receptors grouped into three clusters on either side of the head. Here we show that the peripheral sensory receptors of these two groups of species employ fundamentally different strategies for coding electric communication signals. Species that can discriminate variation in EOD waveforms have receptors that fire a precisely timed action potential in response to each EOD. These receptors use two distinct temporal codes for electric communication signals. Differences in spike times between receptors on opposite sides of the body encode EOD duration, and differences in spike times within the same receptor encode interpulse intervals. In addition, spiking receptors are most sensitive to stimulus frequencies within one octave of the peak power frequencies of conspecific EODs. In contrast, species that cannot detect EOD waveform variation have receptors with spontaneously oscillating (up to $2.2 \mathrm{kHz}$ ) potentials that do not generate action potentials. These receptors respond to electric pulses with oscillatory phase resets. The difference in phase reset between receptors on opposite sides of the body is $\sim 180^{\circ}$ and does not vary with pulse duration. These receptors respond with increased oscillation amplitudes for interpulse intervals near their intrinsic oscillation periods $(\sim 0.5-1.0 \mathrm{~ms})$, which are much shorter than the minimum intervals $(\sim 10 \mathrm{~ms})$ produced by individual signaling fish. Furthermore, oscillating receptors are most sensitive to stimulus frequencies almost two octaves below the peak power frequency of conspecific EODs. This mismatch between receptor sensitivity and signal frequencies, coupled with the receptors' enhanced responses to intervals below those produced by single fish, suggest that these receptors are not tuned to any feature of individual conspecific signals. To test the hypothesis that oscillating receptors are specialized for detecting group communication signals, we recorded the signaling activity of a group of 38 interacting fish and found that the inter-EOD intervals fell within the range of the oscillating receptors' maximum sensitivity. The functional consequences of encoding electric communication signals into phase and amplitude modulations of oscillatory receptors are not yet well understood. Future experiments will address how variation in receptor physiology may relate to differences in behavior, absolute sensitivity, and directional sensitivity.

\section{The Central Role of Time in Establishing Connections in Development and in Evolution}

\author{
Charvet $\mathrm{CJ}^{a}$, Sherwood CC ${ }^{b}$, Takahashi $E^{a}$ \\ aDivision of Newborn Medicine, Boston Children's Hospital,

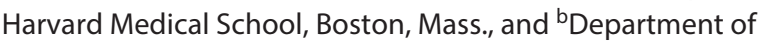 \\ Anthropology, George Washington University, \\ Washington, D.C., USA \\ charvetcj@gmail.com
}

The 'large equals well-connected' hypothesis states that structures that become disproportionately enlarged and mature for an extended period of time (e.g., frontal cortex) should project to more targets than they did ancestrally. The 'large equals well-connected' hypothesis is grounded in development wherein structures that develop for an extended period of time may have more time to compete for targets with other structures. In development, terminal neurogenesis occurs earlier in the frontal cortex than in more caudal regions yet frontal cortex maturation (e.g., myelination) is protracted relative to more caudal regions. That is, the frontal cortex has more time devoted to forming connections. However, it is not clear what the protracted developmental maturation of the frontal cortex actually entails for its connectivity patterns. Very little is known about the basic developmental sequence of frontal cortex tract maturation. We investigate the timing of tract maturation in the frontal cortex of the cat (Felis catus). To that end, we use DSI (diffusion spectrum MR imaging) tractography of cat brains ranging in age from post-natal (P) day 0 to 100. We examine the development of intra- and inter-frontal connectivity. We find that at P0 and P35, intra-frontal connections (i.e., $\mathrm{U}$ fibers) are less mature and smaller than intra-cortical connections in more caudal regions. Between $\mathrm{P} 0$ and $\mathrm{P} 35$, tracts connecting the frontal cortex with subcortical structures (e.g., corticospinal tract) become clearly evident. By P100, U fibers in the frontal cortex and somatosensory cortex are larger than in caudally situated cortical regions. The early maturation of cortico-subcortical tracts may provide the frontal cortex with a competitive advantage in targeting subcortical structures and the protracted maturation of the frontal cortex may foster connections within the frontal cortex. This sequence of maturation should lead to systematic changes in connectivity patterns when developmental schedules are stretched or compressed. The frontal cortex integrates incoming information into action via its subcortical targets and the sequence of frontal cortex maturation may preserve this fundamental architecture in evolution.

\section{Descending Modulation of Auditory Processing in the Amphibian Midbrain}

\author{
Farris $\mathrm{H}$, Ponnath $\mathrm{A}$
}

Neuroscience Center, Louisiana State University School of

Medicine, New Orleans, La., USA

hfarri@Isuhsc.edu

Compared to the ascending auditory pathway, there is less understanding of the role of descending circuitry in processing functional signals, including sorting and assigning signals to their sources. Using awake in vivo single unit recordings in frogs, our study addressed this gap by testing whether electrical stimulation of the thalamus modulates auditory excitability in neurons of the midbrain. In initial experiments, two different temporal modes of electrical stimulation tested whether auditory sensitivity could be modulated for periods of time that are longer (i.e., minutes) than functional communication sounds or on a sound-by-sound basis. Following long-term electrical stimulation ( $20 \mathrm{~Hz}$ for $3-10 \mathrm{~s}$ ), excitability (spikes/acoustic stimulus) to free-field noise stimuli decreased $(-32 \%)$ immediately after electrical stimulation and gradually returned over $600 \mathrm{~s}$. In contrast, sound-by-sound electrical stimulation using a single ( 2 -ms) electrical pulse $25 \mathrm{~ms}$ before each 
noise stimulus caused faster and varied forms of modulation: modulation lasted $<2 \mathrm{~s}$ and in different cells excitability either decreased, increased or shifted in latency. Sound-by-sound modulation varied with the type of acoustic stimulus (including male calls), as the probability of modulating sensitivity to three male calls and a broadband noise were not the same. When taken together with a lack of any modulation of spontaneous activity, the data suggest modulation did not alter the general excitability of the auditory cells and thus may be specific for certain stimulus attributes. This was tested directly for stimulus lateral position. For binaural sensitive units, modulation depended on the ear of input, as sound-by-sound electrical stimulation during dichotic acoustic stimulation from ear phones caused asymmetric modulatory effects: e.g., excitability to sounds presented to one ear increased, whereas the other decreased. The result was greater relative differences in binaural sensitivity. Thus, sound-by-sound descending stimulation revealed fast and location-specific modulatory capabilities that appear well suited to the 'on-demand' shifts in auditory attention that may be used to segregate sounds in the natural auditory scene. Lastly, when taken together with in vitro data in frogs and anatomical data across vertebrates showing extensive descending input onto the ascending auditory system, our results suggest that models proposing static auditory filtering may represent exceptions, even in frogs.

\section{No Relative Expansion of the Prefrontal Cortex in Primate and Human Evolution}

\author{
Gabi $M^{a}$, Neves $K^{a}$, Masseron $C^{a}$, Ribeiro $P^{a}$, Ventura-Antunes $L^{a}$, \\ Kaas $\mathrm{JH}^{b}$, Herculano-Houzel $\mathrm{S}^{a}$ \\ aCiências Morfológicas, Univ. Federal do Rio de Janeiro, \\ Rio de Janeiro, Brazil; ' Department of Psychology, Vanderbilt \\ University, Nashville, Tenn., USA \\ marianagabi@gmail.com
}

Human evolution is thought to have involved a selective enlargement of the cerebral cortex, and in particular an expansion of the prefrontal cortex, the cortical region to which some of our remarkable cognitive abilities such as personality expression, planning and decision making, are often attributed. However, this popular notion has been recently challenged [Semendeferi et al.: Nat Neurosci 2002;5:272-276; Barton and Venditti: Proc Nats Acad Sci USA 2013;110:9001-9006]. Our recent work showed that the human cerebral cortex, with only $20 \%$ of all brain neurons despite a relative mass of $82 \%$ of the brain, actually has just as many neurons as would be expected for a primate brain of its size. But the question of how those neurons are distributed within the cerebral cortex, particularly in regard to the distribution of neurons in the prefrontal cortex in comparison to other primates, remains open. Here we determine, for the first time, whether the expansion in absolute volume of the prefrontal cortex (here determined as all cortex anterior to the corpus callosum) occurs with or without an increase in its relative number of neurons, that is, with or without a relative expansion of its processing power compared to the rest of the cortex. To answer this question, we analyzed the cerebral cortex of seven non-human primate species (Saguinus midas, Otol- emur garnetti, Macaca fascicularis, Aotus trivirgatus, Papio papio, Cebus apella, Macaca nemestrina) and one human cortex [Ribeiro et al.: Front Neuroanat 2013;7:28] and determined how neurons are distributed along the anteroposterior axis of the cerebral cortex. We find that the cumulative distribution of neurons by the volume of gray matter is similar in all species, despite a variance in cortical volumes of 40 -fold across species. Most importantly, the prefrontal cortex holds around $8 \%$ of neurons of all cortical regions in all 8 species, including human. The higher concentration of neurons is located in the posterior, occipital regions. These results suggest that cortical expansion in primate evolution happened without an accompanying modification of the distribution of neurons across its surface. Most importantly, cortical expansion in primate (and human) evolution does not exhibit a relative expansion of the number of neurons in the prefrontal cortex. Those $8 \%$ of all cerebral cortical neurons correspond to a much larger absolute number of neurons in human than in other primate brains, which could explain our superior cognitive abilities compared to other primates.

\section{Dynamic Visual Cues Trigger Jaw Opening and Closing by Tiger Beetles during Pursuit of Prey}

Gilbert $C^{a}$, Perkins $M Q^{a}$, Zurek $D B^{a, b}$

aDepartment of Entomology, Cornell University, Ithaca, N.Y., and

bepartment of Biological Sciences, University of Pittsburgh,

Pittsburgh, Pa., USA

cg23@cornell.edu

In dynamic locomotory contexts, visual cues often trigger adaptive behavior by the viewer, yet studies investigating how animals determine impending collisions typically employ either stationary viewers or objects. Here we describe a dynamic situation of visually-guided prey pursuit in which both impending prey contact and escape elicit observable adaptive behaviours in the pursuer, a predatory tiger beetle. We investigated which visual cues independently control both opening and closing of the beetle's jaws during chases of prey dummies. Jaw opening and closing typically occur when prey is within the $60^{\circ}$ field of binocular vision, but not at a specific distance, angular size, or time-to-collision. We show that a sign change in the expansion rate of the target image occurs within about $10 \mathrm{~ms}$ (opening) to $40 \mathrm{~ms}$ (closing) before the jaw movement and provides a trigger value signaling that the beetle is either gaining on the target or that it is getting away. These values are close to the 28-ms lag time we have recently determined for the beetle's locomotor system that controls whole body pursuit of prey. We discuss the 'sloppiness' of the variation in the lag of the behavioural response, especially jaw closing, as an adaptation to uncertainty about target position due to degradation of the target image by motion blur from the fast-running beetle. 


\section{Quantitative Genetics and the Evolution of the Human Brain}

Gómez-Robles $A^{a}$, Hopkins WD ${ }^{b, c}$, Sherwood CC ${ }^{a}$

aDepartment of Anthropology, The George Washington

University, Washington, D.C., b Neuroscience Institute, Georgia

State University, Atlanta, Ga., and 'Division of Developmental

and Cognitive Neuroscience, Yerkes National Primate Research

Center, Atlanta, Ga., USA

aidagomezr@yahoo.es

Chimpanzee brains likely resemble the phenotype of the last common ancestor of hominins and panins. Therefore, unravelling the genetic architecture of chimpanzee brains can help elucidate the evolutionary potential of the brain structure from which modern humans evolved. We address this topic by evaluating genetic variation that is available to selection in chimpanzee brains through evolutionary quantitative genetics, which uses phenotypic information in pedigreed populations to estimate the genetic component of variation from similarities between kin-related individuals. We used structural MRI scans of more than two hundred chimpanzees and geometric morphometric techniques to characterize brain shape (size variation being held constant) through a set of homologous landmarks. Changes in the relative position of these landmarks provided a multivariate context to estimate the phenotypic and genetic covariance matrices. These matrices were compared to assess the evolutionary potential of the genetic architecture of chimpanzee brains and, assuming that this architecture would be similar to that of stem hominins, to evaluate the effect of evolutionary changes observed during early hominin brain evolution. With this aim, we simulated different selective scenarios that mimic particular evolutionary transitions observed during hominin evolution, including a reduction of the visual cortex, parietal reorganization, and an expansion and fronto-lateral projection of the inferior frontal regions. Our results reveal that phenotypic and genetic covariance matrices show clear similarities, and that the main patterns of variation, which are related to the extension of parietal lobes and to the general proportions between brain length and width, have a genetic origin in spite of the extensive plastic variation observed in some brain areas. In addition, we observed that simulated selection gradients elicit responses to selection that are not circumscribed to localized brain regions. However, direct responses in the areas under explicit selection are generally stronger than correlated and delocalized responses to selection induced by genetic correlations in the remaining regions. This result contrasts with studies of cranial integration showing that correlated indirect responses to selection are substantially higher than direct responses to selection and have a primary role in shaping the human craniofacial complex. Our results show that genetic constraints cause moderate adjustments of the brain regions that are not under explicit selection, but not a massive reorganization of the whole brain. In this way, genetic constraints do not override the capacity of particular regions to respond to selection, which may have made the brain of stem hominins markedly evolvable. These results, which are based on intraspecific and size-corrected shape variation, underscore that the evolutionary flexibility of chimpanzee and human brains (and perhaps of other mammalian brains) can be higher than expected on the basis of large-scale interspecific comparisons.

\section{The Eyes Have It: The Visual Guidance of Host Localization and Escape in the Medicinal Leech}

Harley $C M$, Mesce KA

University of Minnesota, St. Paul, Minn., USA

cmharley@umn.edu

Leeches are sanguivores and must locate hosts for their blood meals. During this task, leeches utilize visual and mechanosensory cues that are created when prey animals disturb the surrounding water. These water disturbances focus light, creating specific visual patterns beneath the water which leeches use to localize their prey [Carlton and McVean: Comp Biochem Physiol Comp Physiol 1993;104:273-277; Harley et al.: J Exp Biol 2011;214:3801-3807]. Recent findings indicate that the leech visual system is not used for hunting prey alone; it can also trigger an escape response to noxious UV stimuli [Jellies: J Exp Biol 2014;217:974-985]. Little is known about the leech visual system, let alone how visual stimuli guide the leech to approach or avoid a visual stimulus. Leech head eyes are made up of $\sim 40$ photoreceptors that are arranged in a cone-like shape surrounded by visual pigment [Lasansky and Fuortes: J Cell Biol 1969;42:241-252]. Our anatomical data suggest that connections exist between eyes; perhaps a mechanism for aiding in early visual processing involving contrast enhancement; however, more data are needed to substantiate this idea. Through the use of a novel anatomical-labeling technique, we have also found that the leech's eyes project into the brain such that each eye has a distinctive pattern of terminal arborizations. These patterns are stereotypic across individuals. Preliminary data indicate that eyes located more rostrally project to more anterior regions of the brain, suggesting the potential for a retinotopic map within the leech brain. How specific visual stimuli become integrated into a decision to escape or approach is an important but unknown problem. To address this question, we are studying the different forms of locomotion associated with the detection or avoidance of prey - specifically, whether the leech swims or crawls. Our recent work has shown that leeches crawl more when visual prey stimuli are present; conversely, crawling leeches are better able to localize prey [Harley et al.: J Exp Biol 2013;216:1890-1897]. In contrast, harmful UV stimuli have recently been shown to induce an avoidance response, during which leeches swim away from the stimulus source [Jellies: J Exp Biol 2014;217:974-985]. We predict that prey-like visual stimuli, as compared to UV stimuli and the photoreceptors that are tuned to such wavelengths, may differentially project to brain interneurons that mediate crawling versus swimming. Such interneurons include cell R3b1 and its neighboring neurons, which have been shown to initiate or modulate crawling behavior. Studies to examine UV-related swim initiation are focused on previously identified swim-trigger neurons in the leech brain. Leeches are an extremely diverse group of annelids occupying many niches. What is adaptive for prey detection behavior in one species may not be for another. We have noted that the number and distribution of eyes varies greatly across leech species with different prey preferences. Continued species comparisons may provide us with additional insights into the neural mechanisms underlying the sensory guidance of niche-specific adaptive locomotion and, ultimately, prey detection. 


\section{Drumming 'Thunder Chickens': The Ethology and Neurobiology of a Non-Vocal Courtship Display}

Iwaniuk AN

Department of Neuroscience, University of Lethbridge, Lethbridge, Alta., Canada

andrew.iwaniuk@uleth.ca

The study of the neural basis of courtship behaviors has largely focused upon vocalizations. Courtship can, however, encompass a wide range of non-vocal behaviors in vertebrates and how these signals are perceived and modulated by the nervous system are largely unknown. Among birds, the ruffed grouse (Bonasa umbellus) is an ideal species within which to examine the neural basis of non-vocal courtship. Unlike related galliform species (e.g., turkeys, ptarmigan), male ruffed grouse do not use vocalizations in their courtship whatsoever. Instead, male ruffed grouse flap their wings rapidly to create a low-frequency 'drumming' sound. Drumming is often used for grouse censuses, but until recently, the behavior itself was poorly understood. Bioacoustic analyses revealed that the sound is very low frequency $(<100 \mathrm{~Hz})$ and propagates as an idealized dipole, unlike vocalizations. In addition, the speed at which a male drums and the number of drumbeats in his display varies significantly among individuals. Playback experiments have proven inconclusive in terms of individual recognition, but have demonstrated that ruffed grouse can localize low-frequency acoustic signals. The ability of ruffed grouse to detect low frequencies is not, however, associated with anatomical specializations in the basilar papilla or nuclei along the ascending auditory pathway. Instead, ruffed grouse are similar to all other galliform birds in that more than half of the basilar papilla is dedicated to frequencies $<1 \mathrm{kHz}$. The only difference in the ruffed grouse auditory system appears to be the unique expression of aromatase in field L. In terms of producing the drumming behavior, volumetric analyses of multiple brain regions has shown that males undergo significant seasonal changes in both the basal ganglia and the arcopallium, a premotor region. That is, males that are actively drumming in the spring breeding season have significantly larger basal ganglia and arcopallial volumes than males in the fall, non-breeding season. The large seasonal difference in arcopallium (21\%) parallels changes in pectoral muscle mass and muscle fiber composition, which together suggests that male ruffed grouse undergoes significant remodeling of both the nervous and muscular systems when they are drumming. Similar seasonal changes were not present in other telencephalic regions or the telencephalon as a whole. This is the first evidence of major seasonal changes in the telencephalon of a non-passerine bird species. In addition, it implicates the arcopallium in the production of the drumming display and suggests that seasonal plasticity in the brain and courtship behavior are common features of all forms of avian courtship.

\section{A Diversity of Neuromodulatory Actions and Synaptic Connections Underlies the Evolution of Swimming Behaviors in Nudibranch Sea Slugs}

Katz PS, Gunaratne CA, Sakurai A, Senatore A, Tamvacakis AN

Neuroscience Institute, Georgia State University,

Atlanta, Ga., USA

pkatz@gsu.edu

It is not uncommon for analogous behaviors to have repeatedly evolved within a clade of organisms. Such appears to have been the case with swimming behaviors in the Nudibranchia (Mollusca, Gastropoda, Nudipleura). There are over 5,000 species in this monophyletic clade, yet only about 60 species falling within 15 families swim. There are two primary forms of swimming: dorsalventral (DV) body flexions and left-right (LR) body flexions, which seem to have evolved independently several times. We have studied the neural circuits underlying both forms of swimming in six species, and found that there are notable similarities and differences in how the circuits function. (1) DV and LR swim central pattern generators (CPGs) are comprised of non-overlapping sets of neurons, whereas CPGs for analogous behaviors contain homologous neurons. (2) Homologous neurons can differ substantially in the roles that they play in production of the swimming behaviors even if the behaviors are analogous. (3) Three of the LR swim CPGs differed from each other in participation of neurons in the motor pattern, sensitivity to the cholinergic antagonist curare, synaptic organization, and the necessity of particular neurons for activation of the motor pattern. (4) In one species, Dendronotus iris, curare blocked particular CPG synapses, causing the motor pattern to cease. Replacing the blocked synapses with artificial synapses using the dynamic clamp technique restored rhythmic activity. In contrast, the swim motor pattern in Melibe leonina, whose CPG differs in synaptic organization from that of Dendronotus, was not blocked by curare. Rewiring the Dendronotus swim CPG with artificial dynamic clamp synapses to make it resemble that of Melibe also restored rhythmic activity. This suggests that there are two separate ways for these CPGs to produce rhythmic activity. (5) It was previously shown that DV swimming in both Tritonia diomedea and Pleurobranchaea californica (which serves as an outgroup for the Nudibranchia) can be initiated by and requires serotonin (5-HT). Furthermore, serotonergic neurons in the swim CPGs of both species modulate the synaptic strengths of other CPG neurons, whereas homologous neurons in a non-DV swimmer, Hermissenda crassicornis, lack this 5-HT modulation. We have now sequenced and annotated the central nervous system (CNS) transcriptomes from these species and three others. We have identified the sequences for seven 5-HT receptor subtypes falling in each of the five molluscan 5-HT receptor families. This will allow us to determine whether neuronal differences in 5-HT receptor expression or functionality underlie neuromodulatory and behavioral differences across species. The transcriptomes will also allow us to resolve the phylogeny of Nudibranchia to test the phylogenic hypothesis that the two forms of swimming each arose independently several times. If so, it would suggest that evolution of behavior involves repeated use of homologous neurons in different configurations.

This work was supported by NSF-IOS-1120950. 


\section{Sensory and Motor Responses to Lateral Line Stimulation in Larval Zebrafish}

Liao JC, Akanyeti O, Ballo A, Haehnel-Taguchi M, Levi R

The Whitney Laboratory for Marine Bioscience and Department of Biology, University of Florida, Saint Augustine, Fla., USA

jliao@whitney.ufl.edu

Water flow is detected in fishes using the mechanoreceptive hair cells of the lateral line system, and depends on several factors including the morphology of the neuromasts, hydrodynamics and the physiology of primary afferent neurons. We deflected the hair cell bundles of single neuromasts and evaluated the corresponding afferent neuron activity in 5-day-old larval zebrafish (Danio rerio) using both firing rate and vector strength (a measure of synchronicity to a stimulus) as a response metric. We provided three direct mechanical stimuli; single ramp deflections, pure sine waves and broad frequency pulses. For ramp stimuli, we found that the maximum spike rate of afferent neurons increased sigmoidally with neuromast deflection velocity, with a linear range between 0.1 and $1.0 \mu \mathrm{m} \mathrm{ms}^{-1}$. However, spike rate did not change when a neuromast was deflected below $8 \mu \mathrm{m}$ regardless of deflection velocity. Our findings reveal an unexpected sensitivity in the larval lateral line system: stimulation of a single neuromast could elicit a swimming response which increased in reliability with increasing deflection velocities. For sinusoidal stimuli, we found mainly one type of afferent neuron with band-pass qualities. At low frequencies, spike rate increased linearly up to $30 \mathrm{~Hz}$ and then plateaued. Vector strength peaked at $20 \mathrm{~Hz}$, stabilized at intermediate frequencies, and began to decrease above $60 \mathrm{~Hz}$. Afferent responses changed over the duration of the response, with spike rate decreasing at high but not low frequencies. This suggests that at low stimulation frequencies an afferent neuron can fire indefinitely, while at high frequencies it cannot maintain constant firing. To look at the capacity, reliability, precision and latency during information transmission, we used broadband pulse stimuli. By departing from traditional sine wave stimulation, we were able to show that afferent neurons can theoretically generate a maximum of $\sim 80$ spikes per second, which represents the upper limit of the neurons' capacity. This also allowed us to determine that the reliability of the response decreased after $30 \mathrm{~Hz}$ and that well-timed spikes at low pulse rates lost their precision once the stimulus exceeded $70 \mathrm{~Hz}$. In contrast to sinusoidal stimulation, pulse stimulation additionally revealed that latency was pulse rate dependent. Afferent capacity and reliability gradually decreased over the course of the stimulus. Overall, we discovered that larval afferent neurons have a rich response repertoire, on par with adult fishes, and can distinguish relative frequencies using the different responses of spike rate and vector strength.

\section{Energetics of Active Sensory and Communication Signals in the Weakly Electric Fish Eigenmannia virescens: Organismal, Cellular, and Molecular Perspectives}

\section{Markham MR}

Department of Biology, The University of Oklahoma, Norman, Okla., USA

markham@ou.edu

Active sensory and communication systems such as echolocation in bats are advantageous for many reasons. These systems do not rely on extrinsic energy sources such as sunlight and the organism can control features of the energy source including amplitude, direction, and spectral characteristics. However, these animals must pay the energetic costs associated with producing the sensory carrier signal. Managing the energetic demands of active sensory and communication signals is essential for survival and reproductive fitness, and metabolic stress can compromise both sensory function and social communication. Electric fish generate brief electric organ discharges (EODs) for electrolocation and communication. The EOD is generated by the synchronized action potentials (APs) of the electric organ cells (electrocytes) which generate membrane currents that can exceed 10 microAmperes and incur correspondingly high energetic demands. These energetic demands are driven primarily by the activity of the $\mathrm{Na}^{+} / \mathrm{K}^{+}$ATPase which requires ATP to actively transport $\mathrm{Na}^{+}$and $\mathrm{K}^{+}$across the cell membrane following each AP. The gymnotiform electric fish $E i$ genmannia virescens generates a quasi-sinusoidal electric organ discharge (EOD) at frequencies of 300-600 Hz. These high firing rates further amplify the energetic demand of EOD production, potentially consuming up to $30 \%$ of the animal's energy budget. We have identified physiological adaptations at the organismal, cellular, and molecular levels through which $E$. virescens manages energetic requirements of the EOD during normal conditions and under metabolic stress. E. virescens regulates EOD amplitude on a circadian rhythm to manage energy expenditure. Additionally, under food deprivation E. virescens reduces EOD amplitude then restores signal amplitude following feeding, a change mediated by circulating endocrine factors that regulate electrocyte function. Electrocytes possess several design features that reduce energetic requirements of signal production including the compartmentalization of the ion channels and $\mathrm{Na}^{+} / \mathrm{K}^{+}$ATPases that generate the electrocyte AP and restore ion gradients following the AP. Electrocytes terminate the AP with $\mathrm{Na}^{+}$-activated $\mathrm{K}^{+}$channels rather than voltage-gated $\mathrm{K}+$ channels, and adaptation that further increases energy efficiency of AP generation. 


\section{Bird Brains Have Extraordinarily High Neuron Densities}

Olkowicz $S^{a}$, Kocourek $M^{a}$, Lucan $R^{a}$, Portes $M^{a}$, Herculano-Houzel $S^{b}$, Nemec $P^{a}$

aDepartment of Zoology, Faculty of Science, Charles University in Prague, Czech Republic; ' Instituto de Ciências Biomédicas, Universidade Federal do Rio de Janeiro, Brazil

seweryn.olkowicz@gmail.com

Many birds show remarkable cognitive abilities that can rival those observed in apes, people's closest relatives. With brains the size of only a fraction of that observed in large primates it remains uncertain how birds can accomplish this level of behavioral sophistication. Using the method of isotropic fractionator we investigated neuron and nonneuronal cell numbers in brains of parrots and passerine birds, two avian orders with the most intelligent representatives. We show that in these birds total brain neuron numbers scale approximately linearly with brain size, i.e., the average neuron size shows little increase and neuronal density decreases minimally as brains get larger. In passerines and parrots neuronal densities in the telencephalon always exceed those observed in the cerebral cortex of primates by a factor of 2-8. As a result, the numbers of telencephalic neurons in the brains of the largest of examined birds (raven, kea and macaw) equal or exceed those observed in the cerebral cortex of mediumsized monkeys. The avian cerebellum features neuronal densities similar or higher to those found in primates. In contrast to the latter, however, in birds the relative size of cerebellum decreases with brain size, therefore cerebellar neurons make a smaller proportion of total brain neurons the larger the bird's brain. With increasing neuronal proportion contained in the telencephalon, in the macaw brain almost $80 \%$ of all brain neurons is contained in cerebral hemispheres, while only $20 \%$ populates the cerebellum, a condition reversed to what is found in mammals. Finally, for all examined brain structures, the densities of nonneuronal cells remain constant regardless of brain size, a finding congruent with data from mammals. Our results strongly suggest that high neuronal numbers and hence high brain's computational capacity underpin the behavioral and cognitive complexity reported for parrots and passerine birds.

\section{Brain Scaling and Allometric Variation of Sensory Brain Regions in Lampreys (Petromyzontiformes)}

\author{
Salas $C A^{a}$, Yopak KE ${ }^{a}$, Hart $N S^{a}$, Nazari $H^{c}$, \\ Dominguez-Dominguez $\mathrm{O}^{d}$, Gill HS ${ }^{b}$, Potter $I C^{b}$, Collin SPa
}

aNeuroecology Group, School of Animal Biology and UWA

Oceans Institute, The University of Western Australia,

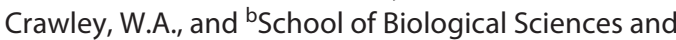

Biotechnology, Murdoch University, Murdoch, W.A., Australia;

'Marine Biology Department, The National University of

Iran, Tehran, Iran; ${ }^{\mathrm{d}}$ Aquatic Biology Laboratory, Universidad

Michoacana de San Nicolas de Hidalgo, Michoacan, Mexico

correodesalas@gmail.com, carlos.salas@ research.uwa.edu.au

Extant lampreys represent the earliest vertebrates and are therefore of great interest for the study of brain evolution and the ancestral condition of the vertebrate brain. Additionally, due to their protracted lifestyle, these fishes represent an excellent model for understanding the role ecological factors play in the development of the brain in a diversity of habitats. However, little is known about the ontogenetic or phylogenetic variability of their nervous system. We investigated the changes in brain size and brain structure size during ontogeny in representatives of all three families of lampreys and were interested to reveal whether there were any differences in brain organization associated with a stage of the life cycle or additional ecological, with implications for specialization of individual sensory modalities. Body mass and brain mass were obtained from 55 individuals from 17 species at each of the five phases of the life cycle, representing 33 independent data points for ammocoetes or larvae $(n=9)$, metamorphosing individuals $(\mathrm{n}=3)$, downstream migrants $(\mathrm{n}=8)$, upstream migrants $(\mathrm{n}=4)$ and spawning adults $(n=8)$. We found that the brain mass increased with body mass according to the allometric relationship $y=0.48 x+1.02\left(R^{2}=0.59, n=33\right.$; slope significance at $\left.p=0.001\right)$. Results from an analysis of variance (ANOVA) revealed that body size had a significantly positive effect on brain size $(\mathrm{p}=0.001)$, which was similar across the different life history stages. A posthoc Tukey analysis showed that ammocoetes had significantly smaller brains than individuals at post-metamorphic stages $(\mathrm{p}<$ 0.05 ), whereas we found no significant differences in brain size between individuals after metamorphosis. Volumes of the olfactory bulbs, the telencephalic lobes, the pineal organ, the optic tecta, the octavo-trigeminal region (V-VIII nerve), and the gustatory area (IX-XII nerve) were approximated using the idealized halfellipsoid/ellipsoid method. To account for the phylogenetic relatedness between species, phylogenetic generalized least-squares (pGLS) models of evolutionary change were also constructed to test for correlates of brain evolution. Akaike's information criteria (AICs) were used to identify the most parsimonious model, which showed that, for some major brain regions, ecological factors such as habitat, diet, and parasitism exerted a significant influence on brain structure size. Remarkably, the best-fit models for olfactory bulb size included parasitism and diet as factors, which was consequently confirmed by an analysis of covariance (ANCOVA), where parasitic species have a higher rate of development of the olfactory bulbs compared to non-parasitic species. Based on our results, we predict that animals at each life history stage experience a wide range of environmental cues within their primary habitat, thus influencing different sensory systems, which may be reflected in changes in brain organization throughout ontogeny. This work offers a unique opportunity to understand how phylogenetic constraints and environmental signals influence the development of the brain in a basal vertebrate. 


\section{Spatial Memory of Food-Hoarding Birds: Cued or Free Recall?}

Smulders TV, Male LH, Read JCA

Centre for Behaviour and Evolution, Newcastle University, Newcastle upon Tyne, UK

tom.smulders@ncl.ac.uk

Scatter-hoarding birds have become a textbook example of adaptive brain specialization. Hiding food in many thousands of locations protects the birds from catastrophic loss of their food reserves, but also faces them with the challenge of retrieving these items later. One potential solution to this problem is to have an adaptively specialized spatial memory system. Indeed, the hippocampal formation, which is involved in spatial memory, is larger in scatter-hoarding birds than in their non-hoarding relatives, suggesting such an adaptive specialization.

However, the nature of this adaptive specialization is not well understood. Can they remember more locations than non-hoarders? Can they retain the information for longer? Is the spatial information more precise? The answer to these questions may be different for different species, as some bury their food (e.g. jays), while others hide it in crevices in their natural foraging niches (e.g. titmice).

Titmice do not just use memory to retrieve their food, but also to decide where to hide new items. This allows them to avoid generating spatial clumps, further reducing the odds of a catastrophic loss. They move through their home range and hoard or retrieve food nearby to where they are at any point in time. Therefore, rather than having to freely recall the locations, they could be cued by the view of the hoarding site itself. Using a technique inspired by recognition memory research in humans, we explore whether coal tits (Periparus ater) use free and/or cued recall when retrieving memories of previously hoarded food items. Understanding how they retrieve their memories will allow us to better investigate how selection has acted on their memory systems in the future.

\section{The Costs of Extreme Encephalization: Bigger Brains Result in Increased Energetic Demand and Reduced Hypoxia Tolerance in Weakly Electric African Fishes}

Sukhum K, Freiler M, Wang R, Carlson BA

Department of Biology, Washington University in St. Louis, St. Louis, Mo., USA

kvsukhum@wustl.edu

A large brain offers several advantages including an increased ability to adapt to new environments, forage for food, and develop social networks. However, brain tissue is energetically costly. So how do animals balance the costs of extreme encephalization? Multiple hypotheses have been suggested for how a species could support an increase in brain tissue, including decreasing the size of other energetically expensive tissues or increasing overall energy consumption. Here, we show that in the mormyrid family of elec- tric fishes, there is not a trade-off in organ size, but instead largebrained fish have increased basal metabolic rates. We also show that an increase in brain size and energy demand comes with decreased hypoxia tolerance in large-brained fish. Weakly electric African fishes, the mormyrids, are an ideal system to compare a wide range of closely related species that display extreme variation in brain size. The expensive tissue trade-off hypothesis predicts that with an increase in brain tissue, there will be a decrease in the size of another expensive organ's tissue. We compared organ masses of the brain, liver, heart, GI tract, kidney, and gonads in 38 species. We did a log-log regression of the species-average mass of each organ against the species-average body size across species, and then took the residuals of each individual across species with respect to these regression lines. This method allowed us to control for similarity in traits due to phylogeny using phylogenetic generalized least squares of the residuals. With or without correcting for phylogenetic nonindependence, we found no correlation between relative brain size and the relative size of any other organ. Thus for mormyrids, there does not seem to have been an expensive organ trade-off related to the evolution of their large brains. We then used closed tank respirometry to compare basal metabolic rates. Based on the anatomy, our hypothesis was that large-brained fish have larger basal metabolic rates than closely related small-brained fish. Indeed, we found that large-brained fish had a significantly higher oxygen consumption rate than small-brained fish. This shows that an overall increase in energetic requirements accompanies having a large brain. We are currently researching how having a large brain and higher energetic requirements affects hypoxia tolerance. In their native habitat and throughout their evolutionary history, mormyrids have encountered low oxygen environments. With a large-brain being so metabolically costly, our hypothesis is that large-brained fish will be more sensitive to hypoxia than small-brained fish. We used sodium sulfite to progressively lower the free oxygen in water and observed large- and small-brained fish's electric organ discharges and behavior. Our preliminary data shows that large-brained fish are less able to tolerate low oxygen environments, whereas smallbrained fish can tolerate lower oxygen levels for a longer period. Our results suggest that increased energetic demand and reduced hypoxia tolerance are important costs of increased encephalization in mormyrids and indicate that variation in brain size may impact the geographical distributions of species due to environmental variation in oxygen availability.

\section{Hominin Evolution: Greater Control of the Visual Percept by the Prefrontal Cortex Evolved as a Result of Selective Pressure from Immobile, Camouflaged Predator}

Vyshedskiy A

Boston University, Boston, Mass., USA

theandreyv@gmail.com

Immobile, camouflaged predators may have acted as the primary selective pressure operating on primates to expand their visual system and brain volume. The Snake Detection theory notes 
that primates exposed to greater predation by venomous snakes acquired a better visual system compared to primates not exposed to venomous snakes [Isbell: The Fruit, the Tree, and the Serpent. Harvard University Press, 2009]. The Mental Synthesis theory extends the Snake Detection theory to the evolution of hominins. The theory observes that hominins foraging in the savanna were exposed to an even greater selective pressure from immobile predators partially hidden by vegetation. While their chimpanzee-like ancestors who dwelled primarily in the treetop canopies were safe from most ground-dwelling predators, hominins venturing into the savanna were facing many new camouflaged and immobile predators including stalking big cats, sabertooths, and hyenas. Unable to fight off the bigger and faster predators, the feeble hominins had only one option for survival: long-distance recognition and avoidance of the stalking predators. Consistent with this additional selective pressure, modern humans evolved to detect immobile visual targets an order of magnitude faster than chimpanzees [Matsuno: Behav Brain Res 2006;172:219] and integrate local immobile elements into a bigger shape significantly better than chimpanzees [Fagot: Anim Cogn 2001;4:171-177]. However, an even more dramatic result of this selective pressure on the visual system may have been the development of the prefrontal cortex' (PFC) ability to voluntarily control the visual percept. The PFC plays an active role in the visual processing of ambiguous stimuli [Windmann: J Cogn Neurosci 2006;18:456-471]. Furthermore, fMRI and lesion studies implicate the PFC in all tasks that rely on active manipulation of visual images into new combinations. The Mental Synthesis theory posits that under the selective pressure from immobile predators, the PFC evolved greater top-down voluntary control of the visual percept. The evolving improvement in control of the visual precept is evident in the ever-increasing sophistication of the stone tools manufactured by hominins. The Homo habilis visual system was getting better at voluntarily excluding neurons representing the surrounding vegetation to reveal a partially hidden predator and also gained the ability to voluntarily exclude neurons representing flakes from the rest of the neuronal ensemble in order to reveal the mental template of the Mode One chopper within. Additional selection further improved the ability of Homo erectus to detect immobile predators and enhanced its voluntary control over its mental image, resulting in manufacturing of more sophisticated Mode Two stone tools. Finally, we, Homo sapiens, are capable of the greatest voluntary control of our mental image: we can imagine anything we want. It is possible that a single evolutionary driving force - predation by hard-to-detect, immobile predators - acting for over 70 million years on the primate visual system, created enough evolutionary pressure to acquire the human brain, much of which is dedicated to processing visual information in the context of our current goals. [The abstract was compiled from 'On The Origin Of The Human Mind', 2nd ed.] A free download of the complete monograph is available: www.mobilereference.com/mind.

\section{Is Bigger Always Better? Developing Quantitative Measures of Cognitive Ability in Early Vertebrates}

\author{
Yopak KE ${ }^{a}$, Heel $K^{b}$, Goh $G^{a, c}$, Northcutt $R G^{d}$, Collin SPa
}

aschool of Animal Biology and the UWA Oceans Institute, University of Western Australia, Crawley, W.A., 'S School of Pathology and Laboratory Medicine, University of Western Australia, Crawley, W.A., and 'School of Anatomy, Physiology and Human Biology, University of Western Australia,

Crawley, W.A., Australia; dDepartment of Neurosciences and

Scripps Institution of Oceanography, University of California San

Diego, San Diego, Calif., USA

kara.yopak@uwa.edu.au

Relationships between the body, brain, and major brain regions have traditionally been used to infer cognitive abilities across all vertebrates, providing vital information about life history traits, behavior and even 'intelligence'. Indeed, we have shown that broad variability in the size and complexity of major brain areas, including the olfactory bulbs, telencephalon, diencephalon, optic tectum, tegmentum, cerebellum, and medulla oblongata, is highly correlated with habitat and/or specific behavior patterns in both bony and cartilaginous fishes. However, new neuronal scaling rules based on a method of accurately assessing the number of neurons in the brain in mammals, isotropic fractionation [see Herculano-Houzel and Lent: J Neurosci 2005;25:2518-2521], suggest that brain mass may be a poor predictor of cognitive ability and enhanced associative function. Here, we present the first application of this technique in fishes, using the Port Jackson shark, Heterodontus portusjacksoni, and the barramundi, Lates calcarifer, as model species. The total number of neurons (as compared to non-neuronal glia) was measured in the brain and its component parts using the isotropic fractionation method. Counts made on cell nuclei were automated via flow cytometry and concordance between automated and manual counting methods was also tested using a haemocytometer. We present detailed strategies for the optimization of brain homogenization, neuronal staining, calibration, imaging, and quantification of brain cells in fishes. Based on preliminary results, we suggest the isotropic fractionation method, coupled with flow cytometry, serves as an effective tool to quantify neuronal scaling in early vertebrates. Further, we present data on total brain cell counts in our representative fish species in the context of currently available mammalian data, and discuss the potential for conservation of neuronal scaling across vertebrates. These data will pave the way for future work to assess whether the number of neurons within the major brain regions show a linear relationship or reveals differential rates of addition in relation to predicting higher cognitive abilities and/or more complex behavioral repertoires in fishes, with implications for how 'intelligence' has evolved across vertebrates. 